injections, and they thus sum up the conclusions to which they have arrived: 1. Recurrence of the disease is less frequent with subcutaneous than with internal treatment. 2. Calomel is preferable to the sublimate and other salts of mercury which have been tried until now, on account of the less gravity and frequency of local and general accidents. 3. Injections with calomcl should be performed on the external and central surface of the arm. 4. The syringe should be introduced with precision into the subcutaneons cellular tissue, and care should be taken that the point be not fixed into the thickness of the derm. 5 . The best vehicle for the calomel is bydrate of gum-arabic. 6. The quantity of calomel used must vary betwcen ten and twenty centigrammes (about two to four grains). 7. Painting with collodion is very effective. 8. There should be an interval of at least ten days betwcen every two injections. 9. T.he injections should bc discontinued if the first two produce little or no amendment. -Lancet, Nov. 23, 1872.

\title{
OPHTHAIMOLOGY.
}

55. Treatment of Strumous Ophthalmia. -Mr. Henry Power, Senior Ophthalmic Surgeon to St. Bartholomew's Hospital, finds that gencral treatment is by no means sufficient to cure the patient, and amongst the many local remedies lie has used, he gives the palm to atropia, in a two or four grain solution. Pagenstecher's yellow ointment, and calomel. With one or the other of these, most cases, he says, may bc cured.

Cases, however, occasionally occur in which all these plans of treatment fail; and the question comes, what must now be tried? It is then that I claim attention to the value of extract of belladonna given internally. I have repeatedly found that it rapidly diminishes the intolerance of light, and by its power of relieving the spasm of the muscles closing the lids, cnables the child to obtain an amount of bencfit from air and exercise that was previously impossible. I can entertain no doubt that its good cffects are attributable to its action as a stimulant upon the sympathetic system of nerves, and through this upon the smaller vessels. It is further of usc in doing away with the necessity for purgatives, as cven in small quantities it acts efficiently in clearing the bowels. I have usually prescribed it in doses of one-eighth to one-quarter of a grain. It is perhaps scarcely necessary to add that, as it is a potent remedy, its effects must be watched, and its administration should be suspended as soon as the child complains of thirst, or when the rapidity of the pulse is observed to be increasing. I have only noticed these symptoms in one or two instances. I have also found tle extract of belladonna serviceable in cases where the affection was rather a limited lieratitis than phlyctenular opbthalmia; that is, in which a small segment of the cornea was hazy and vascular near the margin, even though the intolerance of light may not have been very intense. I consider the seton, thongh recommended by so good an authority as Mr. Bader, a pis aller; and find the treatment mentioned by Dr. Swanzy as practised by $V$ on Gräle, though it was originally suggested by Juingken, of dipping the whole head for a few seconds in cold water, not persistent in its effects.- The Practitioner, Oct. 1872.

56. Evulsion of the Iris.-Mr. W. H. Folken states (Brit. Med. Journ., Oct. 5,1872 ) that, while a man, at. 42 , was pulling at a piece of wood, a portion of it suddenly flew up, striking him in the right cye, making a wound in the outer and lower edge of the cornea. The anterior chamber had some blood in it, and chemosis was setting in; the eye was too painful at the time to allow of minute examination. Mr. F. at once applied atropia, and employed antiphlogistic treatment.

When all inflammation had subsided, and the corncal wound had healed, the iris was found to be completely torn away, a piece of the size of about two pins' heads only remaining at the corner of the wound. There was for a long time 
great intolerance of light; which, however, has gradually subsided, and the patient is now able to beal any moderate amount of light. At the present time, the lens is perfectly clear. If anything, the eye is slightly contracted. On closing the other eye he can see, but cannot discern distinctly.

When tested with Jäger's types he conld read nothing, but, looking through a circular bole, one-sixteenth of au inch in diameter, in a piece of dark paper, he could read Jigger No. 19. With a test-spectacle (No.9) covered with the same piece of paper, he could read No. 16. When the hole in the paper was enlarged to the eighth of an inch he could not distinguish anything; and with neither aperture did any other spectacles snit him.

\section{MIDWIFERY AND GYN ACOLOGY.}

57. Recession of the Fatal Head after Extraction from the Vagina.-Dr. Tabutrau communicated to the Dublin Obstctrical Society ( $J$ une 15 ) the following case of this. In 1859 , he was called to a woman, æt. 22 , in her first confinement. She had, when he first saw her, been thirty hours in labour, under the carc of a self-taught midwife. He found a face presentation with the chin to the pubis, the head low in the pelvis, and the parts fully dilated. After waiting some time and seeing no progress, he determined on applying the forceps. This he did without any difficulty, and in about five minutes be delivered the head, the chin resting bclow the pubic arch. In three minutes afterwards he found the hearl had entirely receded within the ragina. Dr. Tabuteau subsequcntly effected delivery, without difficulty, of a stillborn child. The recovery of the woman was rapid and complete._Dublin Journ. Med. Sci., Nov. 1872.

58. Treatment of some Forms of Extra-Uterine Pregnancy.-Dr. Meadows read a papcr before the Obstetrical Society of London (Nov. 6, 1872) on this subject. Relating a case of the so-called ventral variety, he referred to some remarks made by him, and published in the last yolume of the 'Transactions, in which he adrocated more frcquent resort to the operation of gastrotomy in certain cases of extra-uterine gestation, the object of the operation being to anticipatc, and so to a vert, the almost inevitably fatal rupture of the cyst, and death from hemorrbage. In the former communication the suggestion was also made that, with a view to prevent one of thc grcat dangers attcndant upon this operation-viz., hemorrhage-the placenta should not be removed, but shonld be left to undergo the slow but sure process of atrophy and absorption, it being argued that, as by the removal of the foetus there is no longer any physiological necessity for that structure, its removal by atrophy might fairly be reckoned on. 'T'he present communication was based upon anotlier case of socalled ventral pregnancy, which, coming under the author's care during his absence, had been seen by two of his colleagues at the Hospital for Womcn, Dr. Squarey and Mr. Scott, and a living child at about the seventh month was removed. 'I'he attempt, however, to remove the placenta, which was situate deep down in the pclvis, and the loetal cyst was attended by such frightful hemorrhage that the patient succumbed in a few hours. Dr. Meadows now contended that in all cases where the diagnosis of a living and a viable child could be made out, gastrotomy ought to be performed; and that in order to facilitate the operation, and at the same time to diminish its danger, both the placenta and the containing eyst should be left in situ, spccial care being taken not to detach any portion of the placenta. In this way the operation might be reduced to a very simple process, and the chief care should be, first to extract a living child, and then to do as little damagc as possible to the remaining structures, thus preventing hemorrhage, and enabling them afterwards to undergo the process of atrophy and absorption, just as occurs in some cases where, no operution having bcen performed, the fotus has died, been mummified as it were, and finally been absorbed. 